\title{
Thermodynamics of the Solid Solutions of Isomorphic Substances
}

\section{Allakhverdov GR* and Retivov VM}

State Scientific-Research Institute of Chemical Reagents and High Purity Chemical Substances, Russia

\section{Abstract}

The article described the thermodynamics of binary solid solution of isomorphic inorganic compounds and their influence on the co-crystallization of substances from solutions.

ISSN: 2578-0255

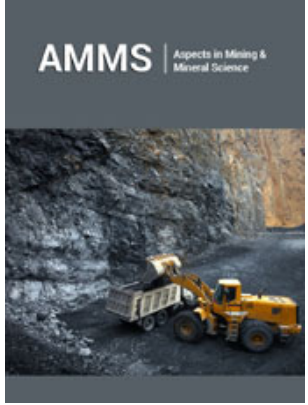

*Corresponding author: G R Allakhverdov, State Scientific- Research Institute of Chemical Reagents and High Purity Chemical Substances- National Research Centre, Kurchatov's Institute, Moscow, Russia

Submission: 此 January 13, 2020

Published: 笽February 26, 2020

Volume 4 - Issue 3

How to cite this article: Allakhverdov GR, Retivov VM. Thermodynamics of the Solid Solutions of Isomorphic Substances. Aspects Min Miner Sci.4(3). AMMS.000590.2020.

DOI: 10.31031/AMMS.2020.04.000590

Copyright@ G R Allakhverdov, This article is distributed under the terms of the Creative Commons Attribution 4.0 International License, which permits unrestricted use and redistribution provided that the original author and source are credited.
Keywords: Activity coefficient; Chemical potential; Co-crystallization coefficient

\section{Introduction}

The study of the conditions and characteristics of the formation of solid solution play an important role in research the processes of formation of mineral and the technology of processing mineral raw materials. The thermodynamics characteristics of the formation of binary solid solutions of isomorphic substances can be calculated using the classical expression for the crystal lattice energy associated with determination of the Coulomb potential and repulse potential in a form acceptable for both components. At the same time this problem can be solved in a more convenient way, using general relations characterizing the dependence of thermodynamics functions on the volume of the system, which leads to an adequate description of the experimental data.

\section{Theory}

Let us consider binary solid solutions of substitution when an impurity component is embedded in the crystal lattice of the main component without the formation of defects. In addition, we use the so-called quasi-harmonic approximation where it is assumed that the vibrations of atoms around the middle equilibrium positions correspond to vibrations of simple harmonic oscillators and the potential energy has a minimum value in these positions. Quantum theory says that the possible levels of the oscillator are defined as

$$
\varepsilon_{n}=\varepsilon_{0}+\left(n_{i}+\frac{1}{2}\right) h \omega(1)
$$

where $\varepsilon_{0}$ is the potential energy in equilibrium, $\mathrm{h}$ is the Plank constant, $\omega$ is the vibrational frequency and $n_{i}$ are integers from zero to infinity. Summing Eq.(1) over all states from the lowest vibrational level at $\mathrm{n}_{\mathrm{i}}=0$, we can determine the partition function [1]

$$
Z=\sum_{0}^{n} e^{-n h \omega / k T}=\left(1-e^{-n h \omega / k T}\right)^{-1}
$$

where $\mathrm{T}$ is a temperature and $\mathrm{k}$ is the Boltzmann constant $(\mathrm{h} \omega / \mathrm{k}$ is the Einstein temperature). The total free Helmholtz crystal energy for $3 v \mathrm{~N}$ oscillators, where $\mathrm{N}$ is the number of atoms and $v$ is a stoichiometric coefficient, can be determined as

$$
A=U-3 v N \ln Z=U+3 v N \ln \left(1-e^{-n h \omega / k T}\right)
$$

where $U=3 v N\left(\varepsilon_{0}+\frac{1}{2} h \omega\right)$ is the energy of the fundamental quantum state, which includes the energy of zero vibrations of atoms $1 / 2 \mathrm{~h} \omega$. At high temperatures we can expansion of the expression

$$
\ln \left(1-e^{-n h \omega / k T}\right) \approx \ln \left(\frac{h \omega}{k T}-\frac{1}{2}\left(\frac{h \omega}{k T}\right)^{2}+\ldots .\right) \approx \ln \frac{h \omega}{k T}-\frac{1}{2} \frac{h \omega}{k T}+\ldots .
$$

and convert Eq.(3) to the form of classical energy

$$
A=U_{0}+3 v N k T \ln \frac{h \omega}{k T}(5)
$$


where $U_{0}=3 v N \varepsilon_{0}$ is the potential energy when all atoms are in equilibrium. Differentiating Eq. (5), we can determine the pressure

$$
P=-\left(\frac{\partial A}{\partial V}\right)_{T}=-\left(\frac{\partial U_{0}}{\partial V}\right)_{T}+\frac{3 v N k T}{V} g(6)
$$

where $\mathrm{V}$ is the volume of system and $\mathrm{g}$ is the Gruneisen constant is the same for all normal vibrations [2]

$$
g=-\frac{d \ln \omega}{d \ln V}(7)
$$

Eq.(7) shows that the vibrational frequency varies depending on the volume as

$$
\frac{\omega_{i}}{\omega_{0}}=\left(\frac{V_{0}}{V_{i}}\right)^{g}(8)
$$

where $\omega_{i}$ is the frequency when the volume is $V_{i}$ and $\omega_{0}$ is the frequency when the volume is $\mathrm{V}_{0}$. The volume of a solid solution, according Retgers rule, can be represented as

$$
V_{0}=y_{1} V_{1}+y_{2} V_{2}(9)
$$

where $\mathrm{y}_{1}, \mathrm{y}_{2}$ are the mole fractions of the components $\left(\mathrm{y}_{1}+\mathrm{y}_{2}=1\right)$ and $V_{1}, V_{2}$ are the volumes of pure components having the common atom in its structures. When a common atom is surrounded by atoms of various species, this atom is shifted towards the atom having a high charge density. It leads to a local change in volume [3]

$$
V_{01}=V_{0}-\lambda\left(V_{2}-V_{1}\right) ; V_{02}=V_{0}+\lambda\left(V_{2}-V_{1}\right)
$$

where $\lambda$ is the value characterizing the displacement of atoms from ideal crystallographic positions in solid solution, subject to equality $\frac{1}{2}\left(V_{01}+V_{02}\right)=V_{0}$.

Differentiating Eq.(5), we can determine the entropy of system

$$
S=-\left(\frac{\partial A}{\partial T}\right)_{V}=-3 v N k \ln \frac{h \omega}{k T}+3 v N k(11)
$$

The change in entropy during the formation of a solid solution associated with a change in the vibrational frequencies of the atoms can be defined as

$$
\Delta S_{v}=S_{0}-y_{1} S_{1}-y_{2} S_{2}
$$

where the subscript " 1 ", " 2 " and "0" refer to pure components and the solid solution, respectively. Substitution Eq.(11) into Eq.(12), we have

$$
\Delta S_{v}=3 v N k \ln \frac{\omega_{1}^{y_{1}} \omega_{2}^{y_{2}}}{\omega_{0}}
$$

Given the different configurations of the surrounding of the common atom by different or identical species of atoms, for example, halogen atoms around the potassium atom in a solid solution $\mathrm{KCl}-$ $\mathrm{KBr}$, the average vibrational frequency can be represented in the form

$$
\omega_{0}=\omega_{11}^{W_{11}} \omega_{12}^{W_{12}} \omega_{21}^{W_{21}} \omega_{22}^{W_{22}}
$$

where $\omega_{\mathrm{ii}}, \omega_{\mathrm{ij}}$ are the frequencies corresponding to the surrounding of the common atom by identical and different species of atoms and $\mathrm{W}_{\mathrm{ii}}, \mathrm{W}_{\mathrm{ij}}$ are probabilities of these combinations

$$
W_{11}=y_{1}^{2} ; W_{12}=W_{21}=y_{1} y_{2} ; W_{22}=y_{2}^{2}
$$

The mechanical theory of vibrations shows that in discrete systems waves with a frequency exceeding a certain final value cannot propagate. For a selected pair of atoms this value can be determined as [2]

$$
2 \pi \omega=\left(2 \frac{c}{M}\right)^{1 / 2}
$$

where $\mathrm{C}$ is the force constant and $\mathrm{M}$ is the reduced mass of atoms. Using Eq.(16), the vibrational frequencies for various configurations can be expressed as

$$
\begin{gathered}
2 \pi \omega_{11}=\left[2 C_{0}\left(\frac{1}{M_{1}}+\frac{1}{M_{0}}\right)\right]^{1 / 2} \\
2 \pi \omega_{12}=\left[2 C_{01}\left(\frac{2}{M_{1}+M_{2}}+\frac{1}{M_{0}}\right)\right]^{1 / 2} \\
2 \pi \omega_{21}=\left[2 C_{02}\left(\frac{2}{M_{1}+M_{2}}+\frac{1}{M_{0}}\right)\right]^{1 / 2} \\
2 \pi \omega_{22}=\left[2 C_{0}\left(\frac{1}{M_{2}}+\frac{1}{M_{0}}\right)\right]^{1 / 2}
\end{gathered}
$$

where $M_{1}, M_{2}$ are the masses of replaced atoms and $M_{0}$ is the mass of common atom (or a group of atoms for complex compounds). For each of the individual components, we can also write

$$
2 \pi \omega_{i}=\left[2 C_{i}\left(\frac{1}{M_{i}}+\frac{1}{M_{0}}\right)\right]^{1 / 2}
$$

Combining Eq.(8) and Eq.(16), we can determine the ratio

$$
\left(\frac{c_{0}}{c_{i}}\right)^{1 / 2}=\left(\frac{V_{i}}{V_{0}}\right)^{g} ;\left(\frac{c_{0 i}}{c_{i}}\right)^{1 / 2}=\left(\frac{V_{i}}{V_{0 i}}\right)^{g}
$$

Then, using Eq.(17)-(22), the average frequency of atomic vibration in a solid solution according Eq.(14) can be determined as [3]

$$
\ln \omega_{0}=y_{1} \ln \omega_{1}+y_{2} \ln \omega_{2}-y_{1} y_{2} j-y_{1} y_{2} p
$$

$$
\begin{aligned}
& \text { where } j=\frac{1}{2} \ln \frac{\left(M_{1}+M_{2}\right)^{2}\left(M_{1}+M_{0}\right)\left(M_{1}+M_{0}\right)}{M_{1} M_{2}\left(M_{1}+M_{2}+2 M_{0}\right)^{2}} \\
& \text { and } \quad p=g\left(\frac{y_{1}}{y_{2}} \ln \frac{V_{0}}{V_{1}}+\ln \frac{V_{01}}{V_{1}}+\frac{y_{2}}{y_{1}} \ln \frac{V_{0}}{V_{2}}+\ln \frac{V_{02}}{V_{2}}\right)
\end{aligned}
$$

Using Eq.(9), (10) and expansion $\ln (1+x)=x$, we have

$$
p=g(1-\lambda) \frac{\left(V_{2}-V_{1}\right)^{2}}{V_{1} V_{2}}
$$

Here, as a first approximation, we can put $\lambda=1 / 2$, whence

$$
p=\frac{1}{2} g \frac{\left(V_{2}-V_{1}\right)^{2}}{V_{1} V_{2}}
$$

Substitution Eq.(23) into Eq.(13), we have

$$
\Delta S_{v}=3 v N k y_{1} y_{2} q
$$

where $q=j+p$.

A complete change of entropy during solid solution formation can be defined as

$$
\Delta S=-R\left(y_{1} \ln y_{1}+y_{2} \ln y_{2}\right)+3 v R y_{1} y_{2} q
$$

where the first term is the entropy of ideal mixing $S_{\text {id }}$ and $R$ is the gas constant $\mathrm{R}=\mathrm{Nk}$, where $\mathrm{N}$ is the Avogadro number. 
At high temperature (here we consider an approximation where the remaining terms of expansion (4) can be neglected) the total energy of a solid can be determined according to Eq.(5) and Eq.(11) as

$$
U=A+T S=U_{0}+3 v R T
$$

where the last term is the same for both the solid solution and the components forming it, so the change in energy during the formation of a solid solution can be represented as

$$
\Delta U=U_{0}\left(V_{0}\right)-y_{1} U_{1}\left(V_{1}\right)-y_{2} U_{2}\left(V_{2}\right)
$$

where the functional dependence of energy on volume is emphasized. Function $\mathrm{U}_{0}\left(\mathrm{~V}_{0}\right)$ can be represented as a hybrid function of the original components [3]

$$
U_{0}\left(V_{0}\right)=y_{1} U_{1}\left(V_{0}\right)+y_{2} U_{2}\left(V_{0}\right)
$$

Take into account the probabilities of various configurations, Eq.(32) should be written as

$$
U_{0}\left(V_{0}\right)=W_{11} U_{1}\left(V_{0}\right)+W_{12} U_{1}\left(V_{01}\right)+W_{22} U_{2}\left(V_{0}\right)+W_{21} U_{2}\left(V_{02}\right)
$$

where $\mathrm{V}_{01}$ and $\mathrm{V}_{02}$ are defined by Eq.(10). Substituting Eq.(33) into Eq.(31), we have

$\Delta U=y_{1}^{2}\left(U_{1}\left(V_{0}\right)-U_{1}\left(V_{1}\right)\right)+y_{1} y_{2}\left(U_{1}\left(V_{01}\right)-U_{1}\left(V_{1}\right)\right)+y_{2}^{2}\left(U_{2}\left(V_{0}\right)-U_{2}\left(V_{2}\right)\right)+y_{1} y_{2}\left(U_{2}\left(V_{02}\right)-U_{2}\left(V_{0}\right)\right)$ (34)

The functions $\mathrm{U}_{\mathrm{i}}\left(\mathrm{V}_{0}\right)$ and $\mathrm{U}_{\mathrm{i}}\left(\mathrm{V}_{0 \mathrm{i}}\right)$ for each of the components can be expanded into Taylor series keeping only the quadratic terms in it, since $d U / d V=0$ in equilibrium. Then, using Eq.(9), we can write, for example, for the first component

$$
\begin{gathered}
U_{1}\left(V_{0}\right)=U_{1}\left(V_{1}\right)+\frac{1}{2} y_{2}^{2} u_{1}\left(V_{2}-V_{1}\right)^{2} \\
U_{1}\left(V_{01}\right)=U_{1}\left(V_{1}\right)+\frac{1}{2}\left(y_{2}-\lambda\right)^{2} u_{1}\left(V_{2}-V_{1}\right)^{2}
\end{gathered}
$$

where

$$
u_{1}=\frac{d^{2} U_{1}\left(V_{1}\right)}{d V_{1}^{2}}(37)
$$

Similar expressions when replacing subscripts can be written for another component. Further, combining Eq.(34)-(36), we have

$$
\Delta U=\frac{1}{2} y_{1} y_{2}\left(V_{2}-V_{1}\right)^{2}\left[u_{1}\left(y_{2}-2 \lambda y_{2}+\lambda^{2}\right)+u_{2}\left(y_{1}-2 \lambda y_{1}+\lambda^{2}\right)\right] \text { (38) }
$$

Using the condition of minimum energy $\frac{d \Delta U}{d \lambda}=0$, we have

$$
\lambda=\frac{y_{1} u_{2}+y_{2} u_{1}}{u_{1}+u_{2}}(39)
$$

Substituting Eq.(39) into Eq.(38), we obtain

$$
\Delta U=\frac{1}{2} y_{1} y_{2}\left(V_{2}-V_{1}\right)^{2} \frac{\left(y_{1} u_{2}+y_{2} u_{1}\right)\left(y_{1} u_{1}+y_{2} u_{2}\right)}{u_{1}+u_{2}}(40)
$$

Turning to a change in enthalpy according to Eq.(6), we have

$$
\Delta H=\Delta U+\Delta(P V)=\Delta U+3 v R T\left(g_{0}-y_{1} g_{1}-y_{2} g_{2}\right)
$$

For isomorphic substances, which have a similar properties, the last term of Eq.(41) can be neglected, therefore, we can put $\Delta \mathrm{H}=\Delta \mathrm{U}$. Than the Eq.(40) can be transformed to the form [3]

$$
\Delta H=\frac{1}{2} y_{1} y_{2} \Omega
$$

where the value of $\Omega$ can be determined in good approximation as

$$
\Omega=\frac{1}{4}\left(V_{2}-V_{1}\right)^{2}\left(u_{1}+u_{2}\right)
$$

Finally, the change of Gibbs energy during the formation of a solid solution, according Eq.(29) and (42), can be represented as

$$
\Delta G=\frac{1}{2} y_{1} y_{2} \Omega+R T\left(y_{1} \ln y_{1}+y_{2} \ln y_{2}\right)-3 v R T y_{1} y_{2} q \text { (44) }
$$

Further using condition $d \Delta G /\left(d_{i}\right)=0$, we can determine the critical temperature of the decomposition of the solid solution

$$
T_{C}=\frac{2 \Delta H_{e q}}{R\left(1+\frac{3}{2} v p\right)}(45)
$$

where $\Delta \mathrm{H}_{\mathrm{eq}}$ corresponds to the equal molar ratio of components.

\section{Discussion}

One of the main controversial issues in the theory of the formation of inorganic solid solution is the existence of ordered structures [4,5]. To solve this problem, we consider a partially ordered solution, where probabilities of surrounding a common atom with the same and different atoms can be represented as [4]

$$
\begin{gathered}
w_{11}^{*}=y_{1}\left(y_{1}-y_{2} \sigma\right) ; w_{22}^{*}=y_{2}\left(y_{2}-y_{1} \sigma\right) ; \\
w_{12}^{*}=w_{21}^{*}=y_{1} y_{2}(1+\sigma)(46)
\end{gathered}
$$

where $\sigma$ is a degree of local order. Then the entropy of mixing can be defined as

$S_{\sigma}=-R\left(w_{12}^{*} \ln w_{12}^{*}+w_{22}^{*} \ln w_{22}^{*}+2 w_{12}^{*} \ln w_{12}^{*}-y_{1} \ln y_{1}-y_{2} \ln y_{2}\right)$

and Eq.(30) can be transformed to

$U_{0}\left(V_{0}\right)=y_{1}\left(y_{1}-y_{2} \sigma\right) U_{1}\left(V_{0}\right)+y_{2}\left(y_{2}-y_{1} \sigma\right) U_{2}\left(V_{0}\right)+y_{1} y_{2}(1+\sigma)\left[U_{1}\left(V_{01}\right)+U_{2}\left(V_{02}\right)\right]$

By transformations similar to Eq.(31)-(34), the change enthalpy during the formation of a solid solution can be expressed as

$$
\Delta H_{\sigma}=y_{1} y_{2} \Omega[1-\Lambda(1+\sigma)](49)
$$

where

$$
\Lambda=\frac{u_{1} \lambda\left(2 y_{2}-\lambda\right)+u_{1} \lambda\left(2 y_{1}-\lambda\right)}{y_{1} u_{2}+y_{2} u_{1}}(50)
$$

Using the condition of minimum Gibbs energy $d \Delta G / d \sigma=0$, we have [6]

$$
\frac{y_{1} y_{2}(1+\sigma)^{2}}{\left(y_{1}-y_{2} \sigma\right)\left(y_{2}-y_{1} \sigma\right)}=\exp \left[\frac{\Omega}{R T}\left(\Lambda+(1+\sigma) \frac{d \Lambda}{d \sigma}\right)\right]
$$

Further, to simplify the view of formulas, we consider, without loss of generality, a solid solution with an equal molar ratio of components. Then the solution of differential equation (51) can be represented as

$$
\Lambda=\frac{1}{1+\sigma}\left[\Lambda_{0}+\frac{R T}{\Omega}(2(1-\sigma) \ln (1-\sigma)+2(1+\sigma) \ln (1+\sigma))\right] \text { (52) }
$$

The integration constant $\Lambda_{0}$ can be determined from the boundary condition - for $\sigma=0$ and $\lambda=1 / 2$, according to Eq.(50), we have $\Lambda_{0}=1 / 2$. Substituting Eq.(52) into Eq.(49) and to make a comparison with Eq.(42), the change enthalpy when ordering the solution can be determined as 


$$
\delta H=\Delta H_{s}-\Delta H=-\frac{1}{2} R T[(1-\sigma) \ln (1-\sigma)+(1+\sigma) \ln (1+\sigma)]
$$

The change in entropy under these conditions can be defined as $\delta S=S_{\sigma}-S_{i d}$ and using Eq.(47) to determine the change in Gibbs energy as $\delta G=\delta H-T \delta S=0$. Thus, the coupling Eq.(52) provides a minimum Gibbs energy for any conjugate values of $\lambda$ and $\sigma$. The most convenient calculation is at $\sigma=0$ using Eq.(44). Note that in this case entropy takes the maximum value.

For practical calculations, we define $\mathrm{u}_{\mathrm{i}}$ as

$$
u_{i}=\frac{1}{\beta_{i} V_{i}}\left[1+T\left(\frac{\partial \ln \beta}{\partial T}\right)_{V}\right](5
$$

where $\beta$ is the compressibility and the last term is about $5-10 \%$ of the basic value [3]. Thus, the value of $\mathrm{V}, \beta$ and $\mathrm{g}$ for a pure components are required to calculate all thermodynamics characteristics of the formation of solid solutions. This data and calculation results according Eq.(42) are given in Table $1 \& 2$. Figure 1 shows that the change in enthalpy during the formation of a solid solution is close to a symmetric parabolic shape.

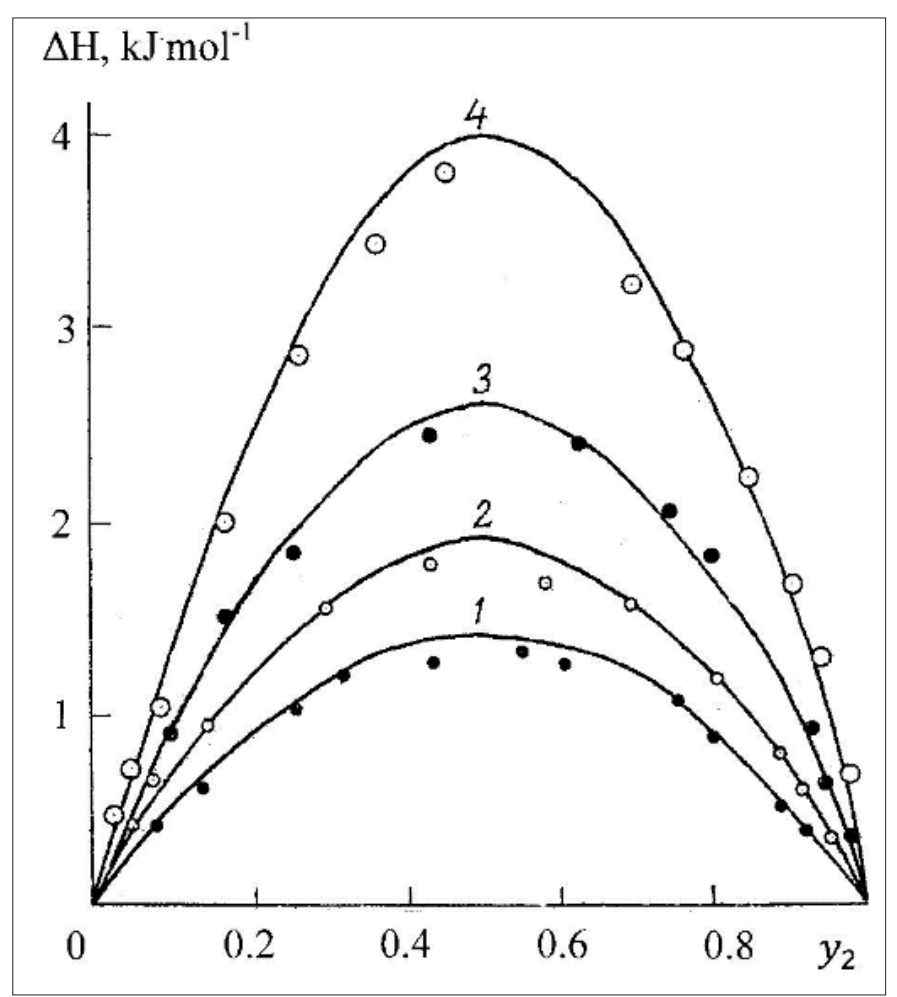

Figure 1: Enthalpy of formation of solid solutions. Calculated curve and experimental dots [7].

1: NaCl-NaBr, 2: KBr-KI, 3: NaBr-NaI, 4: NaBr-KBr.

Table 1: The properties of the individual components at 298K [3].

\begin{tabular}{|c|c|c|c|}
\hline Substance & $\mathbf{V}, \mathbf{c m}^{\mathbf{3}} \mathbf{m o l}^{\mathbf{1}}$ & $\mathbf{1 0}^{\mathbf{1 1}} \boldsymbol{\beta}, \mathbf{P a}^{\mathbf{1}}$ & $\mathbf{g}$ \\
\hline $\mathrm{NaCl}$ & 27.02 & 4.11 & 1.62 \\
\hline $\mathrm{KCl}$ & 37.54 & 5.62 & 1.58 \\
\hline $\mathrm{RbCl}$ & 43.13 & 6.4 & 1.53 \\
\hline $\mathrm{NaBr}$ & 32.17 & 5 & 1.66 \\
\hline $\mathrm{KBr}$ & 43.17 & 6.59 & 1.5 \\
\hline $\mathrm{RbBr}$ & 49.29 & 7.66 & 1.68 \\
\hline $\mathrm{NaI}$ & 40.86 & 6.67 & 1.63 \\
\hline $\mathrm{KI}$ & 53.12 & 8.31 & 1.73 \\
\hline $\mathrm{RbI}$ & 59.59 & 9.28 & 1.07 \\
\hline $\mathrm{K}_{2} \mathrm{SO}_{4}$ & 65.34 & 5.23 & 1.02 \\
\hline $\mathrm{Rb}_{2} \mathrm{SO}_{4}$ & 73.21 & 5.53 & 1.15 \\
\hline $\mathrm{Cs}_{2} \mathrm{SO}_{4}$ & 84.94 & 6.02 & \\
\hline
\end{tabular}


Table 2: Thermodynamics data of solid solutions of equal molar composition at 298K. In parentheses are experimental values, Ref. [3].

\begin{tabular}{|c|c|c|c|c|c|}
\hline System & $\Delta S_{v^{\prime}}$ J.mol ${ }^{-1} K^{-1}$ & $\Delta \mathrm{S}, \mathrm{J} \cdot \mathrm{mol}^{-1} \mathbf{K}^{-1}$ & $\Delta \mathrm{H}, \mathrm{J} \cdot \mathrm{mol}^{-1}$ & $\mathbf{T}_{c}, \mathbf{K}$ & $\Delta \mathrm{G}, \mathrm{J}^{\mathrm{mol}} \mathrm{mol}^{-1}$ \\
\hline \multirow[t]{2}{*}{$\mathrm{NaCl}-\mathrm{NaBr}$} & 0.81 & 6.58 & 1414 & 284 & -555 \\
\hline & & & (1339) & $(300)$ & \\
\hline \multirow[t]{2}{*}{$\mathrm{NaBr}-\mathrm{NaI}$} & 0.68 & 6.44 & 2613 & 500 & 694 \\
\hline & & & $(2406)$ & (538) & \\
\hline \multirow[t]{2}{*}{$\mathrm{NaCl}-\mathrm{KCl}$} & 1.43 & 7.19 & 4719 & 844 & 2576 \\
\hline & & $(6.94)$ & (4393) & $(773)$ & \\
\hline \multirow[t]{2}{*}{$\mathrm{NaBr}-\mathrm{KBr}$} & 1.26 & 7.02 & 4026 & 670 & 1934 \\
\hline & & & $(3724)$ & $(668)$ & \\
\hline \multirow[t]{2}{*}{$\mathrm{NaI-KI}$} & 1.1 & 6.86 & 3127 & 498 & 1083 \\
\hline & & & (2615) & (518) & \\
\hline \multirow[t]{2}{*}{$\mathrm{KCl}-\mathrm{KBr}$} & 0.86 & 6.62 & 915 & 183 & -1057 \\
\hline & & $(6.23)$ & (929) & & $(-933)$ \\
\hline \multirow[t]{2}{*}{$\mathrm{KBr}-\mathrm{KI}$} & 0.79 & 6.55 & 1913 & 387 & -39 \\
\hline & & & (1830) & & \\
\hline \multirow[t]{2}{*}{$\mathrm{KCl}-\mathrm{RbCl}$} & 0.76 & 6.52 & 913 & 186 & -1030 \\
\hline & & $(6.32)$ & $(908)$ & & $(-971)$ \\
\hline \multirow[t]{2}{*}{$\mathrm{KBr}-\mathrm{RbBr}$} & 0.93 & 6.69 & 807 & 159 & -1189 \\
\hline & & $(6.49)$ & $(749)$ & & $(-1184)$ \\
\hline \multirow[t]{2}{*}{$\mathrm{KI}-\mathrm{RbI}$} & 0.96 & 6.72 & 608 & 119 & -1395 \\
\hline & & $(6.57)$ & $(669)$ & & $(-1289)$ \\
\hline \multirow[t]{2}{*}{$\mathrm{RbCl}-\mathrm{RbBr}$} & 1.03 & 6.79 & 823 & 158 & -1200 \\
\hline & & $(6.73)$ & $(795)$ & & $(-1217)$ \\
\hline $\mathrm{K}_{2} \mathrm{SO}_{4}-\mathrm{Rb}_{2} \mathrm{SO}_{4}$ & 0.53 & 6.29 & 1045 & 199 & -829 \\
\hline
\end{tabular}

Using Eq.(44),we can define the excess mixing energy

$$
\Delta G^{E}=\Delta G-\Delta G_{i d}=\frac{1}{2} y_{1} y_{2} \Omega-3 v R T y_{1} y_{2} q
$$

and further, using well-known ratio, the chemical potential of the component (Gibbs partial energy), for example, with a subscript "2"

$$
\Delta \mu_{2}=\Delta G^{E}-y_{1} \frac{\partial \Delta G^{E}}{\partial y_{1}}=\left(1-y_{2}\right)^{2}\left(\frac{1}{2} \Omega-3 v R T q\right)(56)
$$

This value determines the difference in the chemical potential of the component in a real and ideal solid solution, from where the activity coefficient of the component can be determined as

$$
\ln f_{2}=\frac{\Delta \mu_{2}}{R T}=\left(1-y_{2}\right)^{2} E(57)
$$

where

$$
E=\left(\frac{\Omega}{2 R T}-3 v q\right)(58)
$$

Such solutions can be called quasi-regular, their difference from regular solutions consist in taking into account the vibrational component of entropy.
Co-crystallization coefficient of any component, for example, with subscript "2", can be represented as [8]

$$
D_{2}=\frac{y_{2} x_{1}}{y_{1} x_{2}}=\frac{a_{1 L}^{0}}{a_{2 L}^{0}} \frac{\gamma_{2} f_{1}}{\gamma_{1} f_{2}}
$$

where $\mathrm{x}_{1}$ and $\mathrm{x}_{2}$ are relative concentrations of components of mixed liquid solution $(\mathrm{x}=\mathrm{vm} / \mathrm{W}, \mathrm{m}$ is molality, $\mathrm{W}$ is number of moles of solvent per $1 \mathrm{~kg}$ ), $\gamma_{1}$ and $\gamma_{2}$ are activity coefficients, $a_{1 L}^{0}$ and $a_{2 L}^{0}$ are activity of components of saturated binary solutions, In differential form Eq.(59) can be written as

$$
d \ln D_{2}=d \ln L+d \ln \frac{f_{1}}{f_{2}}(60)
$$

where

$$
L=\frac{a_{1 L}^{0}}{a_{2 L}^{0}} \frac{\gamma_{2}}{\gamma_{1}}
$$


The activity coefficient of component in a mixed solution, according Ref. [8], can be represented as

$$
\gamma_{i}=\frac{a_{i}^{*}}{\sum x_{i}}(62)
$$

where $a_{i}^{*}$ is the activity of component in an isopiestic binary solution. Using Eq.(61), (62) and the boundary conditions, we can determine the derivative function $d \ln \frac{L}{d y}$ as the change in value $\ln L$ in the range of concentrations from $\mathrm{y}_{2}=0$ to $\mathrm{y}_{2}=1$ :

$$
L^{\prime}=\frac{d \ln L}{d y_{2}} \approx \frac{\Delta \ln L}{\Delta y_{2}}=\ln \frac{a_{1 L}^{0} a_{2 L}^{*}}{a_{2 L}^{0} a_{1 L}^{*}}
$$

where $a_{i} L^{*}$ is the activity of a component in its binary solution, which is isopiestic to the saturated solution of another component. Using Gibbs-Duhem equation for solid phase

$$
y_{1} d \ln a_{1 s}+y_{2} d \ln a_{2 s}=y_{1} d \ln f_{1}+y_{2} d \ln f_{2}=0
$$

where $a_{i s}=y_{i} f_{i}$ is activity of component in solid phase, and Eq.(57), we have

$$
\frac{d \ln \frac{f_{1}}{f_{2}}}{d y_{2}}=2 E(64)
$$

Substituting Eq.(63) and (64) into Eq.(60), we obtain to linear dependence of the co-crystallization coefficient from composition (Figure 2)

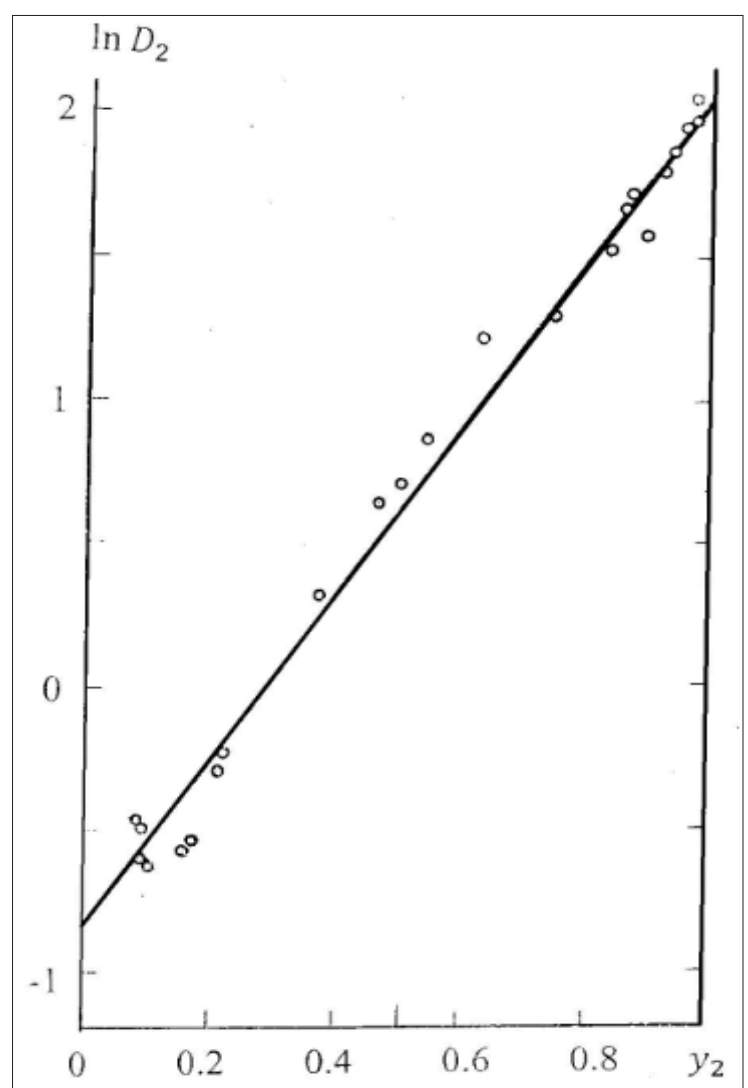

Figure 2: Co-crystallization coefficient in the system $\mathrm{Rb}_{2} \mathrm{SO}_{4}-\mathrm{K}_{2} \mathrm{SO}_{4}-\mathrm{H}_{2} \mathrm{O}$. Calculated line and experimental dots [9].

$$
\ln D_{2}=\ln D_{2}^{*}+\left(L^{\prime}+2 E\right) y_{2}
$$

For other component, we can use definition $\ln D_{1}=-\ln D_{2}$. Thus, according Eq.(65), the co-crystallization coefficient can be defined for any ratio of components, whence it follows that the minimum value of the co-crystallization coefficient $\mathrm{D}_{2}{ }^{*}$ takes in the limiting case when $y_{2} \rightarrow 0$. In this method the values $\mathrm{a}_{i}^{*}$ can be determined by comparing the osmotic coefficients in binary solutions of both components, using extrapolation of data for one of components to the field of supersaturated solutions $[10,11]$. Therefore, as a first approximation it is possible to apply a simpler method of calculation of co-crystallization coefficients using the ratio $\frac{\gamma_{1}}{\gamma_{2}} \approx 1$ for a mixed solutions, which is confirmed for compounds with similar properties [12]. Then, using Eq.(57) and (59), we have

$$
\ln D_{2}=\ln \frac{a_{1 L}^{0}}{a_{2 L}^{0}}-\left(1-2 y_{2}\right) E
$$

The results of calculation according to Eq.(66) show its acceptability for the estimation of co-crystallization coefficient (Table 3); [13]. 
Table 3: Co-crystallization coefficient in ternary water-salt systems at 298K. Experimental data D2* from Ref. [13]. Impurity component is the second in writing.

\begin{tabular}{|c|c|c|c|c|}
\hline $\begin{array}{l}\text { System } \\
\left(+\mathrm{H}_{2} \mathrm{O}\right)\end{array}$ & $\frac{a_{1 L}^{0}}{a_{2 L}^{0}}$ & $\Delta \mu_{2}^{*} \mathbf{k J} \cdot \mathbf{m o l}^{-1}$ & $D_{2}^{*}$ (calc.) & $D_{2}^{*}$ (meas.) \\
\hline $\mathrm{NaCl}-\mathrm{NaBr}$ & 0.33 & 4.69 & $5.10^{-2}$ & $5.10^{-2}$ \\
\hline $\mathrm{NaCl}-\mathrm{KCl}$ & 2.173 & 17.17 & $2.10^{-3}$ & $5.10^{-3}$ \\
\hline $\mathrm{KCl}-\mathrm{NaCl}$ & 0.461 & 17.17 & $4.10^{-4}$ & $6.10^{-4}$ \\
\hline $\mathrm{KCl}-\mathrm{KBr}$ & 0.772 & 2.64 & 0.27 & 0.26 \\
\hline $\mathrm{KCl}-\mathrm{RbCl}$ & 0.637 & 2.74 & 0.21 & 0.21 \\
\hline $\mathrm{KBr}-\mathrm{KCl}$ & 1.295 & 2.64 & 0.45 & 0.45 \\
\hline $\mathrm{KBr}-\mathrm{KI}$ & 0.491 & 6.71 & $3.10^{-2}$ & $4.10^{-2}$ \\
\hline $\mathrm{KBr}-\mathrm{RbBr}$ & 1.013 & 2.12 & 0.43 & 0.42 \\
\hline KI-NaI & 0.064 & 11.2 & $7.10^{-4}$ & $1.10^{-3}$ \\
\hline $\mathrm{KI}-\mathrm{KBr}$ & 2.037 & 6.71 & 0.14 & 0.45 \\
\hline $\mathrm{KI}-\mathrm{RbI}$ & 1.83 & 1.29 & 1.09 & 1.02 \\
\hline $\mathrm{K}_{2} \mathrm{SO}_{4}-\mathrm{Rb}_{2} \mathrm{SO}_{4}$ & 0.454 & 3.55 & 0.11 & 0.13 \\
\hline $\mathrm{RbCl}-\mathrm{KCl}$ & 1.57 & 2.74 & 0.52 & 0.83 \\
\hline $\mathrm{RbBr}-\mathrm{KBr}$ & 0.987 & 2.13 & 0.42 & 0.55 \\
\hline $\mathrm{RbI}-\mathrm{KI}$ & 0.536 & 1.29 & 0.32 & 0.23 \\
\hline $\mathrm{Rb}_{2} \mathrm{SO}_{4}-\mathrm{K}_{2} \mathrm{SO}_{4}$ & 2.202 & 3.55 & 0.52 & 0.45 \\
\hline
\end{tabular}

\section{Conclusion}

The considered model of the formation of solid solutions allow to determine the thermodynamics parameters of solutions based on the properties of the individual components of solution. This model is also applicable for calculating co-crystallization coefficient in the entire composition range of ternary water-salt systems, including the area of micro-concentration of the impurity component where the co-crystallization coefficient takes a minimum value.

\section{References}

1. Landay LD, Lifshits EM (1995) Statistical physics. Moscow, Russia.

2. Girifalco LA (1973) Statistical physics of materials. NY J Wiley \& Sons, New York, USA

3. Allakhverdov GR (2019) Thermodynamics of solutions and separation of elements during crystallization. Moscow, Russia.

4. Wasastjerna JA (1949) On the theory of the heat formation of solid solutions. Soc Sci Fenn Comm Phys Math 15(3): 13.

5. Hietala J (1963) Heat of formation of the sodium chloride type. Annal Acad Sci Fenn 122: 32.
6. Allakhverdov GR (1980) Thermodynamics of formation of the solid solution. Zh Phys Chem 54(3): 617-621.

7. Lister MW, Meyers NF (1958) Heat of formation of some solid solutions of alkali halides. J Phys Chem 62(2): 145-150.

8. Allakhverdov GR, Zhdanovich OA (2019) Theoretical aspects of obtaining pure inorganic substances during crystallization from solutions. AMMS 3(2): 396-397.

9. Stepin $\mathrm{BD}$, Allakhverdov GR (1976) Thermodynamics of system $\mathrm{K}_{2} \mathrm{SO}_{4}^{-}$ $\mathrm{Rb}_{2} \mathrm{SO}_{4}-\mathrm{H}_{2} \mathrm{O}$ at 298K. Zh Inorg Chem 21(11): 311-315.

10. Allakhverdov GR, Zhdanovich OA (2019) Thermodynamics of the ternary water-salt systems. AMMS 4(3): 491-494.

11. Allakhverdov GR (2019) Thermodynamics of the electrolyte solutions. AMMS 4(1): 465-474.

12. Ratner AG, Makarov LL (1960) Thermodynamics of system KCl-RbCl$\mathrm{H}_{2} \mathrm{O}$ at 298K. Zh Phys Chem 34(11): 2495-2501.

13. Allakhverdov GR, Mikhlin AL (2017) Separation factor for the crystallization of inorganic substances from aqueous solutions. Inorganic Materials 53(5): 525-528. 